\title{
Development of Suitable ISE Measurement Procedures for SI-Traceable Chemical Activity Determination
}

\author{
Daniel Berdat*, Hanspeter Andres, and Samuel Wunderli
}

\begin{abstract}
In clinical chemistry and medical diagnostics, the reliability of the results obtained by numerous testing protocols has a major impact on the critical decision-making processes. Considerable efforts are made by international institutes to promote and give guidance on measurement comparisons and metrological traceability using appropriate certified reference materials. The concentration values or amount of substance contents are usually assigned to chemical analytical results. In contrast, the most relevant quantity is the activity of ions in clinical chemistry. Activity corresponds to the available biologically active part of these components in a mixture. Many measurement methods and instruments currently in use do not differentiate between the total substance concentration and its biologically active component. In physiological solutions, such as blood plasma, the chemical activity makes a clear difference between ion or substrate concentration. Ion selective electrodes (ISE) are widely used to directly measure the activity of ions. Measurement by means of ion-selective electrodes is a standard method in point of care testing units (POCT) for continuous monitoring for e.g. haemodialysis, cardiac surgery and in intensive care units. A metrological approach to ISE potentiometric measurements is proposed, to determine the activity with their uncertainty. A prerequisite is to develop an activity calibration scale for complex electrolyte solutions with known and traceable ion activities.
\end{abstract}

Keywords: Clinical chemistry $\cdot$ Electrolyte $\cdot$ Ion-selective electrode $\cdot$ Pitzer activity $\cdot$ Potentiometry

\section{Introduction}

Within the growing number of analytical techniques and innovative tools that are being developed nowadays, numerous testing protocols are applied in clinical chemistry and medical diagnostics. The reliability of the results obtained has a major impact on the critical decision-making processes. [1]

For this purpose, considerable efforts are carried out by international institutes to promote and give guidance on measurement comparisons and metrological traceability using appropriate certified reference materials. ${ }^{[2]}$ The legal requirements for products manufactured and marketed in Europe, stated in the 'In Vitro Diagnos-

${ }^{\star}$ Correspondence: Dr. D. Berdat

Federal Office of Metrology METAS

Section Analytical Chemistry

Lindenweg 50

$\mathrm{CH}-3003$ Bern-Wabern

Tel.: +4131323 3111

Fax: + 41313233210

E-Mail: daniel.berdat@metas.ch tic Medical Directive' (98/79/EC), demand that the traceability of values that are assigned to calibrators and control materials must be assured with reference measurement procedures and/or available reference materials of a higher order. ${ }^{[3-5]}$ The Joint Committee for Traceability in Laboratory Medicine (JCTLM), an assembly established by the International Committee of Weights and Measures (CIPM), the International Federation for Clinical Chemistry and Laboratory Medicine (IFCC), and the International Laboratory Accreditation Cooperation (ILAC), recommends specially qualified laboratories and metrological institutions to assign reference values to standards. Accreditation in accordance with ISO 17025 and ISO 15195 is mandatory for the non-metrological laboratories. They disseminate the reference values further in intercomparison campaigns to routine laboratories on an annual basis. ${ }^{[6]}$

By implementing the requirements of specific international standards, laboratories can validate their competence by assessing the reliability of diagnostic tests . ${ }^{[7-9]}$ These standards are defined by a quality management system that includes the handling and storage control of samples and reference materials, the use of diagnostic devices with approved repeatability, the internal quality control and monitoring of analytical procedures, the traceability of experimental results including evaluation of the measurement uncertainty.
The first step of method validation for diagnostic measurement procedures is the definition of the requirements for the intended use, setting the aimed performance characteristics. The factors to be considered for method validation include numerous parameters such as identity, selectivity/specificity, limits of detection, linear working range, precision, repeatability, intra- and interlaboratory reproducibility and uncertainty. An appropriate mathematical model equation of the measurand has to be set up with an equation formed by the input parameters. An uncertainty budget of all known influence parameters has to be established including the possible contributions such as the realisation and definition of the measurand, the corrections of systematic influences and their uncertainties, and all influences that are directly or indirectly functionally related in the model equation. ${ }^{[10]}$ To each contribution a standard uncertainty is assigned, either by statistical means based on experimental data, called Ttype A evaluation, or by scientific computation based on a chosen distribution, called type B. The standard uncertainties of significance are propagated to form the combined uncertainty $u_{c}$ with their sensitivity factors. The different expressions of measurement uncertainties are developed in the Guide to the Expression of Uncertainty in Measurement. [11]

Metrological traceability of national or international standard references relies 
in the experimental comparison of known and unknown materials, each step characterised with values for the measurand and its uncertainties. ${ }^{[12]}$ The measurement results of control materials can then be traced back to a primary reference measurement procedure and/or calibrator through an unbroken chain of comparisons, all having stated measurement uncertainties. ${ }^{[13]}$ The JCTLM has made the decision to develop lists of higher order procedures and materials in a database for laboratory medicine and in vitro diagnostics, in relation to the requirements of the EU-IVD Directive. ${ }^{[14]}$ These materials and/or procedures are prepared and evaluated by international (BIPM, JRC, WHO, IFCC, NCCLS) or national (PTB, SMU, METAS, NIST, etc.) metrological institutions. The complexity of the validation process requires an appropriate planning. Written documents specifying the information obtained from different sources should be prepared, including a validation programme, the experiments to be carried out, records of raw data and their elaboration, evidence of comparison with the requirements, and an approved conformity statement.

A joint research project of the European Metrology Research Programme (EMRP) was proposed to focus on traceable measurements for biospecies and ion activity in clinical chemistry, under the acronym Tracebioactivity. As part of this project, the Federal Office of Metrology (METAS, Switzerland) collaborates on the metrological aspects of activity measurement of essential ionic species and is developing electrochemical methods and infrastructure to set up traceable ion activity measurements.

\section{Experimental}

\subsection{Reagents}

The high purity salts $(\mathrm{NaCl}, \mathrm{KCl}$, $\mathrm{CaCl}_{2} \cdot 2 \mathrm{H}_{2} \mathrm{O}$ and $\left.\mathrm{MgCl}_{2} \cdot 6 \mathrm{H}_{2} \mathrm{O}\right)$ were purchased from MV Laboratories Inc., Frenchtown, NJ, USA). Aqueous solutions were prepared by gravimetry of the characterised salts and dissolving them in ultrapure water from an ELGA Puralab-ultra unit (Labtec, Wohlen, Switzerland).

The ion-selective membranes were supplied from C-CIT GmbH (Wädenswil, Switzerland), containing the following selective ionophores: Sodium ionophore $\mathrm{X}$ : 4-tert-butylcalix[4]arene-tetracetic acid tetraethylester; potassium ionophore I: valinomycin; chloride TDMACl: tridodecylmethylammonium chloride; calcium ionophore I (ETH 1001): diethyl-N,'[(4R,5R)-4,5-dimethyl-1,8-dioxo-3,6-dioxaoctamethylene]-bis(12-methylaminododecanoate); magnesium ionophore VI (ETH 5506): 1,3,5-tris[10-(1-adamantyl)-

Table 1. Selected ionophores in ion-selective membranes

\begin{tabular}{llll} 
Ionophore & Name & Reference & Abbreviation \\
\hline Potassium ionophore I & Valinomycin & Fluka 60403 & $\mathrm{K}^{+}(\mathrm{I})$ \\
Sodium ionophore X & - & Fluka 71747 & $\mathrm{Na}^{+}(\mathrm{X})$ \\
Chloride TDMACI & TDMACl & Fluka 91661 & TDMACl \\
Calcium ionophore I & ETH1001 & Fluka 21192 & $\mathrm{Ca}^{2+}(\mathrm{I})$ \\
Magnesium ionophore VI & ETH5506 & Fluka 63112 & $\mathrm{Mg}^{2+}(\mathrm{VI})$
\end{tabular}

Table 2. Composition of the mixed electrolyte standard solutions

\begin{tabular}{|c|c|c|c|c|c|c|}
\hline \multicolumn{7}{|c|}{ Molality $m$ / (mmol' $\left.\mathbf{k g}^{-1}\right)$} \\
\hline Solution & $\mathrm{Na}^{+}$ & $\mathbf{K}^{+}$ & $\mathrm{Cl}^{-}$ & $\mathrm{Ca}^{2+}$ & $\mathbf{M g}^{2+}$ & I \\
\hline 1 & 129.67 & 4.62 & 136.7 & 0.72 & 0.48 & 136.8 \\
\hline 2 & 140.07 & 3.68 & 147.7 & 1.20 & 0.77 & 148.4 \\
\hline 3 & 149.92 & 2.74 & 158.1 & 1.69 & 1.04 & 159.4 \\
\hline 4 & 134.93 & 3.23 & 140.5 & 0.67 & 0.52 & 140.6 \\
\hline 5 & 135.92 & 3.32 & 141.9 & 0.77 & 0.57 & 142.1 \\
\hline 6 & 136.95 & 3.40 & 143.3 & 0.86 & 0.62 & 143.6 \\
\hline 7 & 137.92 & 3.49 & 144.7 & 0.96 & 0.67 & 145.1 \\
\hline 8 & 138.81 & 3.58 & 145.9 & 1.06 & 0.71 & 146.5 \\
\hline 9 & 139.91 & 3.67 & 147.4 & 1.16 & 0.76 & 148.1 \\
\hline 10 & 139.62 & 3.74 & 147.5 & 1.25 & 0.80 & 148.2 \\
\hline 11 & 141.90 & 3.87 & 150.2 & 1.35 & 0.86 & 151.1 \\
\hline 12 & 142.91 & 3.96 & 151.7 & 1.45 & 0.95 & 152.7 \\
\hline 13 & 144.91 & 4.22 & 154.7 & 1.64 & 1.14 & 156.1 \\
\hline
\end{tabular}

7,9-dioxo-6,10-diazaundecyl]benzene (Table 1).

\subsection{Preparation of Mixed Electro- lyte Reference Standards}

A series of 13 electrolyte reference standard solutions, each containing a different mixture of the $\mathrm{Na}^{+}, \mathrm{K}^{+}, \mathrm{Mg}^{2+}, \mathrm{Ca}^{2+}$ and $\mathrm{Cl}^{-}$ions in physiological concentrations, was prepared according to Table 2. ${ }^{15]}$ The chloride salts were weighed with high precision on a Mettler XP205 balance with a resolution of $10^{-5} \mathrm{~g}$ (maximum load $220 \mathrm{~g}$ ) and the aqueous solutions obtained were weighed on a XP2004S balance (Mettler Toledo, Greifensee, Switzerland) suitable for higher loads $\left(10^{-4} \mathrm{~g}\right.$ resolution, maximum load $2300 \mathrm{~g}$ ). The weighing procedure was followed by recording the display values and the environmental data (ambient temperature, air density, humidity) simultaneously through an ALMEMO 2890-9 data logger (AHLBORN, Holzkirchen, Germany) connected to a LabVIEW software interface. The recorded data was then used to perform a correction for the air buoyancy by implementing the density of each solution measured with a DMA 58 density measuring device (PAAR, Graz, Austria). A purity correction is also mandatory, this way the exact masses and related molalities could be calculated for the ionic content of the standard solutions.

\subsection{Flow-through Device and Potentiometric lon-selective Measuring System}

The cell at METAS used for potentiometric measurements is symbolically represented as follows: ${ }^{[16]}$

Ag $\left|\operatorname{AgCl}_{(\mathrm{s})}\right| \mathrm{KCl}: 3 \mathrm{M} \mathrm{KCl}::$ sample || membrane \| internal filling solution $\left|\mathrm{AgCl}_{(\mathrm{s})}\right| \mathrm{Ag}$

The outer reference is a closed $\mathrm{Ag} / \mathrm{AgCl}$ gel-type electrode supplied by C-CIT (Wädenswil, Switzerland). The gel material is saturated with potassium chloride and protected with a $0.23 \mu \mathrm{m}$ porosity cellulose membrane; the channel in contact with the sample solution has an approximated diameter of $0.8 \mathrm{~mm}$. With the inner gel-type material, a drift of the potential is observed depending on the exposure time with the sample solutions, probably due to polymer changes and/or depletion, as reported in flow-through electrode setups. [17]

The working electrode is composed of an ion-selective membrane and an inner reference electrode of the $\mathrm{Ag} / \mathrm{AgCl}$-type. 
The internal filling electrolyte solutions used were $0.1 \mathrm{M} \mathrm{NaCl}, 0.1 \mathrm{M} \mathrm{KCl}, 0.1 \mathrm{M}$ $\mathrm{CaCl}_{2}$ or $0.1 \mathrm{M} \mathrm{MgCl}_{2}(0.2 \mathrm{M} \mathrm{NaCl}$ for the chloride electrode) saturated with $\mathrm{Ag}^{+}$in accordance with the detected ion species.

Prior to the experiments, the dry liquid membranes were conditioned for 24 hours in a $0.1 \mathrm{M}$ chloride salt solution of the selected ion.

The automated potentiometric flow system designed and built at METAS, depicted in Fig. 1, is an improved version of previously described work. ${ }^{[2]}$ The electric valve drives K-6 (KNAUER, Berlin, Germany) have been adapted and allow us to perform runs of up to 18 solutions sequentially with three switchable valve blocks of six entries. A METAS calibrated potentiometer with six measurement channels (C-CIT) allows us to perform multichannel detections with PFA flow modules in which the membranes are clamped and up to six working electrodes (in our experiments four electrodes were connected). Air temperature, air pressure and air humidity were measured with an ALMEMO 28909 data logger (AHLBORN, Holzkirchen, Germany) connected to METAS calibrated sensors. A flow controller measures the liquid flow of the system (Sensirion CMOSens ASL 1600-20 liquid flow sensor) and in order to prevent the loss of standards or samples by system failure, a max-flow controller (INETRONIC, Zollbrück, Switzerland) is connected at the end of the system to supervise the total flow volume. A bar code reader (Metrologic Instruments Inc., Suzhou, China) coupled with a classification system for chemical compounds and solutions is prepared for sample and standards identification. The potentiometric measurements and environmental data acquisition are performed using a versatile LabVIEW software (Heinz Herren, ISET, Laupen, Switzerland) and measurements are stored using a SQLite database engine.

\subsection{Activity Calculation in Mixed Electrolytes}

The semi-empirical ion-interaction approach for pure and mixed electrolytes used in our work was mainly developed by Pitzer and co-workers.[18,19] Pitzer's semi-empirical theory is able to describe the non-ideal behaviour of the electrolytes up to high ionic strengths in very good agreement with experimental results. The single ion activities of the physiological solutions were calculated using the software PHREEQC version 2.12.1 (David Parkhurst, U.S. Geological Survey) from their gravimetric-related data shown in Table 3 using the Mac Innes convention. ${ }^{[20-23]}$ The Mac Innes convention asserts the equality of the activity coefficients of po-

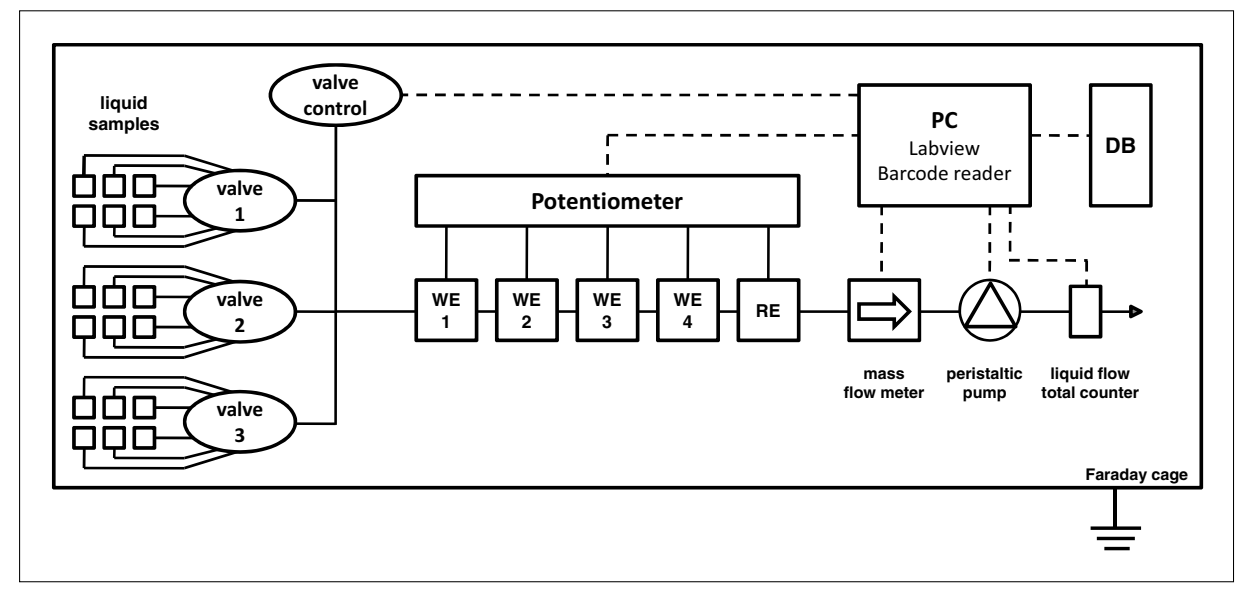

Fig. 1. Schematic view of the automated potentiometric flow system at METAS. Three valves connected each with six sample bottles are piloted by a valve controller. The work electrodes (WE) and reference electrode (RE) are connected to a potentiometer and the data acquisition is performed with a computer equipped with a LabVIEW software. The liquid is pumped through the fluidic system and measured with a mass flow meter. The flow is supervised by a total flow counter to prevent wasting of standard solution in case of system failure. A total volume of $2 \mathrm{~mL}$ is used per cycle of measurement for the 13 standards.

Table 3. Activities of the ionic content in the standard solutions

\section{Activity a / ( $\left.\mathbf{m m o l} \cdot \mathbf{k g}^{-\mathbf{1}}\right)$}

$\begin{array}{cccccc}\text { Solution } & \mathbf{N a}^{+} & \mathbf{K}^{+} & \mathbf{C l}^{-} & \mathbf{C a}^{2+} & \mathbf{M g}^{2+} \\ \mathbf{1} & 99.7 & 3.48 & 101.8 & 0.250 & 0.170 \\ \mathbf{2} & 107.2 & 2.75 & 109.2 & 0.408 & 0.267 \\ \mathbf{3} & 114.3 & 2.04 & 115.9 & 0.563 & 0.357 \\ \mathbf{4} & 103.5 & 2.43 & 104.4 & 0.232 & 0.184 \\ \mathbf{5} & 104.3 & 2.49 & 105.2 & 0.263 & 0.200 \\ \mathbf{6} & 105.1 & 2.55 & 106.2 & 0.296 & 0.218 \\ \mathbf{7} & 105.6 & 2.62 & 107.2 & 0.328 & 0.233 \\ \mathbf{8} & 106.3 & 2.68 & 107.9 & 0.359 & 0.247 \\ \mathbf{9} & 107.1 & 2.75 & 109.0 & 0.394 & 0.265 \\ \mathbf{1 0} & 106.9 & 2.80 & 109.1 & 0.423 & 0.279 \\ \mathbf{1 1} & 108.4 & 2.90 & 110.8 & 0.455 & 0.297 \\ \mathbf{1 2} & 109.2 & 2.95 & 111.7 & 0.490 & 0.328 \\ \mathbf{1 3} & 110.4 & 3.14 & 113.6 & 0.549 & 0.393\end{array}$

tassium and chloride in a pure aqueous solution of potassium chloride of any concentration or content; ${ }^{[24]}$ the activity coefficients of these ions are then assumed to take the same values also in other mixtures of electrolytes of the same ionic strength. The software PHREEQC gives the mean activity coefficient for each ion species and the corresponding single ion activity is calculated using the relation $a_{i}=\gamma_{i} \cdot m_{i}$.

\section{Method to Measure Ion Activities in Complex Electrolyte Mixtures}

\subsection{Preparation of Standards and Potentiometric Measurements}

To create the activity scale for specific ions, two interdependent elements are necessary: Firstly the activity of the desired ion in the complex electrolyte sample solution needs to be known. This value originates from the introduction of the electrolyte molality in the Pitzer model, and is related to the masses of the components. Secondly, the electromotive force has to be measured for each standard solution and with each ion-selective sensor. These two components of the activity scale have to be determined with their corresponding combined measurement uncertainty to achieve comprehensive analytical assays.

\subsection{Mass of Pure Electrolyte Components Forming the Mixture}

The preparation of the standard solutions containing the electrolytes $\mathrm{Na}^{+}, \mathrm{K}^{+}$, $\mathrm{Cl}^{-}, \mathrm{Ca}^{2+}$ and $\mathrm{Mg}^{2+}$ involves the development of a standardised weighing procedure that yields the lowest uncertainty through 
the chain of calculations for the determination of the mass and molality.

The combined uncertainty given with a mass determination incorporates influence factors that originate from the physical and technical specifications of the balance (repeatability, nonlinearity, sensitivity tolerance, and temperature coefficient of the sensitivity) and the effect of air buoyancy.

As described by Reichmuth et al., the value and the combined standard uncertainty of mass determination is expressed in Eqns (1) and (2) where $m$ is the mass of the sample, $u\left(m_{s}\right)$ the combined uncertainty of the mass, $B u$ is the air buoyancy correction factor, $u(B u)$ its corresponding uncertainty, $w$ is the weighing value displayed by the balance and $u(w)$ the standard uncertainty of this value.[25]

$$
m_{s}=B u \cdot w_{s}
$$

$$
\frac{u_{c}\left(m_{s}\right)}{m_{s}}=\sqrt{\left(\frac{u(B u)}{B u}\right)^{2}+\left(\frac{u\left(w_{s}\right)}{w_{s}}\right)^{2}}
$$

\subsection{Potentiometric Measurements}

The challenge is to develop a measuring system that will be able to acquire an EMF signal that, combined with the ion activities, will give a slope as close as possible to the expected ideal Nernstian sensitivity values described for ion-selective electrodes. ${ }^{[26]}$ Membranes for the sensors are chosen according to their least interference. The relationship between the potential $E$ of a measuring electrode circuit and the activity $a_{\mathrm{i}}$ of an ionic species to which it responds is in general mainly governed by the Nernst equation (Eqn. (3))

$$
E=E^{0}+\frac{R \cdot T}{z \cdot F} \cdot \ln \left(\frac{a_{i}}{m^{0}}\right)
$$

where $E^{0}$ is the standard potential, $R$ is the gas constant, $F$ is the Faraday constant, $T$ is the temperature in Kelvin, $z$ is the number of elementary charges of the ion $i$ to be sensed and the unit molality $m^{0}$ $\left(1 \mathrm{~mol} \cdot \mathrm{kg}^{-1}\right)$ creates a pure number as the argument for the logarithm function. Apart from the fixed charge number $z$, the factor before the logarithm in this equation depends only on the temperature $T$. The term $2.303 \cdot(R \cdot T /(z \cdot F))$ is called the slope factor or Nernst sensitivity, and expresses the theoretical change of the potential $E$ of a measuring electrode for a tenfold change in the activity $a_{\mathrm{i}}$ of the sensed ion. Thus for monovalent $(z$ $=1)$ and divalent ions $(z=2)$, at $T=298.15$ $\mathrm{K}$, the calculated Nernst selectivities are respectively $59.2 \mathrm{mV}$ and $29.6 \mathrm{mV}$.
In the presence of other ionic species $j$, most polymeric membranes incorporating ionophores for mono- and divalent ions have shown a behaviour that can be expressed by an extended form of the Nernst equation, the Nikolskij-Eisenman equation (Eqn. (4)).[27,28]

$$
\begin{aligned}
& E_{I}=E_{I}^{0}+E_{J}+ \\
& \frac{R \cdot T}{z_{I} \cdot F} \cdot \ln \left[\frac{a_{I}}{m^{0}}+\sum_{i \neq I}\left(K_{I, i 1}^{p o t}\right)\left(\frac{a_{i 1}}{m^{0}}\right)^{z_{I} / z_{i 1}}\right]
\end{aligned}
$$

where $E_{I}^{0}$ sums up all constant potential differences in the circuit, $E_{J}$ is the liquid junction potential between the reference electrolyte and the sample or standard solution. ${ }^{[29]}$

In our model, Eqn. (4) is also extended by a dynamic term $\kappa$, that represents the response of the ion-selective membrane in function of time, and a drift term $D$ as shown in Eqn. (5):

$$
\begin{aligned}
& E_{I}=E_{I}^{0}+E_{J}+D+ \\
& \kappa \cdot \frac{R \cdot T}{z_{I} \cdot F} \cdot \ln \left[\frac{a_{I}}{m^{0}}+\sum_{i \neq I} K_{I, i 1}^{p o t} \cdot\left(\frac{a_{i 1}}{m^{0}}\right)^{z_{I} / z_{i 1}}\right]
\end{aligned}
$$

The combined uncertainty is dominated by the uncertainties in $E_{J}$ and $\kappa$, whereas the uncertainties resulting from the ion activity coefficients are comparatively small in Eqn. (5). [2] The uncertainty in $E_{J}$ is generally considered as a major source of bias in ISE measurements, whereas the uncertainty of the dynamic term is usually not considered. $[30,31]$

\subsection{The Pitzer Semi-empirical Ion Interaction Model}

The Pitzer model describes the ionic activities as a function of solution ionic strength (long-distance interaction), inter- action terms (short-distance interaction), temperature and pressure. ${ }^{[32]}$

This model mainly consists of a virial equation, the Pitzer parameters are fitted to real measurements. These equations can adequately express the thermodynamic properties of the concentrated electrolyte solution over a wide range of concentrations and temperatures. ${ }^{[33]}$ The Pitzer model, based on a virial expansion, is reduced to a modified form of the Debye-Hückel formula at low ionic strength. ${ }^{[20,34]}$ This virial expansion involves summations over all possible binary and ternary short-range interaction terms, as well as mixing terms.

The equations of Pitzer were derived from the following virial expansion of the excess Gibbs free energy $G^{\text {ex }}$ that is the difference between molal Gibbs free energy and the molal Gibbs free energy of an ideal solution system in which no interactions between ions are postulated (Eqn. (6)).

The symbols in Eqn. (6) are: $m_{w}=$ mass (in $\mathrm{kg}$ ) of solvent water; $m_{i}, m_{j}, \ldots=$ molalities of the subscripted solutes; $\lambda_{i j}\left(I_{\mathrm{m}}\right)=$ binary interaction coefficients; $\mu_{i j k}=$ ternary interactions coefficients.

The factors $\lambda_{i j}\left(I_{m}\right)$ (Eqn. 6b) and $\mu_{i j k}$ (Eqn. 6c) represent the effects of shortrange forces between two ions $\left(\lambda_{i j}\right)$ and three $\left(\mu_{i j k}\right)$ ions. The $\lambda_{i j}$ depend on the ionic strength $I_{m}$, but the $\mu_{i j k}$ are independent of it. Both the $\lambda_{i j}$ and $\mu_{i j k}$ are symmetric, for example $\lambda_{i j}=\lambda_{i j}$ and $\mu_{i j k}=\mu_{i k j}=\mu_{j i k}$. For the interaction between ions and neutral molecules, these coefficients are generally assumed to be constant at a given temperature and independent of the ionic strength $I_{m}$.

In practice the terms $\Theta_{\mathrm{ij}}$ and $\Psi_{\mathrm{ijk}}$ are mostly neglected as their contribution is small.

Starting from Eqns $(6 a, 6 b, 6 c)$, the expressions for the activity coefficient $\gamma_{i}$ of the $i^{\text {th }}$ species are obtained by evaluation of the following partial derivative (Eqn. (7))

$$
\frac{G^{e x}}{m_{w} \cdot R \cdot T}=f\left(I_{m}\right)+\sum_{i} \sum_{j} \lambda_{i j}\left(I_{m}\right) \cdot m_{i} \cdot m_{j}+\sum_{i} \sum_{j} \sum_{k} \mu_{i j k} \cdot m_{i} \cdot m_{j} \cdot m_{k}+\ldots
$$

$$
f\left(I_{m}\right)=-A_{\Phi}(T) \cdot \frac{4 \cdot I_{m}}{b} \cdot \ln \left(1+b \cdot \sqrt{I_{m}}\right) \text { Debye-Hückel term }
$$

$\lambda_{i j}\left(I_{m}\right)= \begin{cases}B_{i j}\left(I_{m}\right) \text { for }\left(z_{i} \cdot z_{j} \neq\left|z_{i} \cdot z_{j}\right| \text { cation - anion dependent on ionic strength } I_{m}\right. \\ \Theta_{i j} \text { for cation - cation, anion - anion, ion - molecule, molecule - molecule }\end{cases}$

$\mu_{i j k}=\left\{\begin{array}{lr}C_{i j} \cdot \sum_{k} m_{k} \cdot\left|z_{k}\right| \text { for }\left(z_{i} \cdot z_{j} \neq\left|z_{i} \cdot z_{j}\right|\right. & \text { cation - cation - anion, cation - anion - anion } \\ \Psi_{i j k} & \text { otherwise }\end{array}\right.$ 


$$
\begin{aligned}
\ln \gamma_{i}=\frac{\partial\left(\frac{G^{e x}}{R \cdot T}\right)}{\partial n_{i}}= & \frac{1}{2} \cdot z_{i}^{2} \cdot f^{\gamma}+2 \cdot \sum_{j} m_{j} \cdot B_{i j}+\sum_{j}\left(\sum_{k} m_{k} \cdot\left|z_{k}\right|\right) \cdot m_{j} \cdot C_{i j}+ \\
& \frac{1}{2} \cdot z_{i}^{2} \cdot \sum_{j} \sum_{k} m_{j} \cdot m_{k} \cdot B_{j k}^{\prime}+\frac{1}{2} \cdot\left|z_{i}\right| \cdot \sum_{j} \sum_{k} m_{j} \cdot m_{k} \cdot C_{j k}
\end{aligned}
$$

$$
f^{\gamma}=\frac{d f\left(I_{m}\right)}{d I_{m}}=-2 \cdot A_{\Phi}\left[\frac{\sqrt{I_{m}}}{1+b \cdot \sqrt{I_{m}}}+\frac{2}{b} \cdot \ln \left(1+b \cdot \sqrt{I_{m}}\right)\right]
$$

$$
\begin{aligned}
& A_{\Phi}=\frac{\ln (10)}{3} \cdot A_{\gamma} \text { with } \\
& A_{\gamma}=\frac{1}{\ln 10} \cdot \sqrt{2 \cdot 1000 \cdot \pi \cdot N_{A} \cdot \frac{d_{w}}{1000}} \cdot\left(\frac{e^{2}}{4 \cdot \pi \cdot \varepsilon_{0} \cdot \varepsilon_{w} \cdot k \cdot T}\right)^{3 / 2}
\end{aligned}
$$

$$
\begin{array}{r}
B_{i j}\left(=\lambda_{i j}\right)=\beta_{i j}^{(0)}+ \\
+\frac{2 \cdot \beta_{i j}^{(1)}}{\alpha_{1}^{2} \cdot I_{m}} \cdot\left\{1-\left(1+\alpha_{1} \cdot \sqrt{I_{m}}\right) \cdot \exp \left(-\alpha_{1} \cdot \sqrt{I_{m}}\right\}\right. \\
\frac{2 \cdot \beta_{i j}^{(2)}}{\alpha_{2}^{2} \cdot I_{m}} \cdot\left\{1-\left(1+\alpha_{2} \cdot \sqrt{I_{m}}\right) \exp \left(-\alpha_{2} \cdot \sqrt{I_{m}}\right)\right\}
\end{array}
$$

$$
\begin{aligned}
B_{i j}^{\prime}= & \frac{2 \cdot \beta_{i j}^{(1)}}{\alpha_{1}^{2} \cdot I_{m}^{2}}\left\{1+\left(1+\alpha_{1} \cdot \sqrt{I_{m}}+0.5 \cdot \alpha_{1}^{2} \cdot I_{m}\right) \exp \left(-\alpha_{1} \cdot \sqrt{I_{m}}\right)\right\} \\
& \frac{2 \cdot \beta_{i j}^{(2)}}{\alpha_{2}^{2} \cdot I_{m}^{2}}\left\{1+\left(1+\alpha_{2} \cdot \sqrt{I_{m}}+0.5 \cdot \alpha_{2}^{2} \cdot I_{m}\right) \exp \left(-\alpha_{2} \cdot \sqrt{I_{m}}\right)\right\}
\end{aligned}
$$

$$
C_{i j}=\frac{C_{i j}^{\phi}}{2 \cdot \sqrt{\left|z_{i} \cdot z_{j}\right|}}
$$

however circumstances where the activity and the concentration are significantly different from each other, and, an approximation with concentrations is not appropriate where activities are required.

\subsection{Potentiometric Signal and \\ Activity Scale \\ 4.1.1 Potentiometric Signal}

The electrochemical response of our ISE flow system for the $\mathrm{K}^{+}$selective membrane is shown in Fig. 2. In this figure, the EMF measurement signal of a sequence of the 13 standard solutions listed in Table 2 is represented versus the measuring time. Each solution is pumped into the system during 90 seconds at a gradual flow rate reaching a maximum of $0.1 \mathrm{~mL} \cdot \mathrm{min}^{-1}$, and the signal is measured in steady state during an hour. In Fig. 2a, the sequence of injection is written above the $\mathrm{x}$ axis, and the signal corresponding to each of the standard solutions is depicted. In this example, only one sequence cycle is shown, however we routinely perform five cycles of measurements in a row, to favour the signal stabilisation over a long period of time. Fig. $2 b$ shows a focus on the signal corresponding to solution 1 , between hour 1 and hour 2: The signal stabilises in only a few minutes, and the subsequent potential drift during an hour of acquisition is estimated to $40 \mu \mathrm{V}$. A major contribution to this drift is the slow variation of the liquid junction potentials at the external reference electrode. With a closer view at the last minutes of the acquisition (Fig. $2 \mathrm{c}$ ), where the signal is the most stable, the noise observed is about $10 \mu \mathrm{V}$.

where $f^{8}$ is defined in Eqn. (7a) and $A_{\Phi}$ and $A_{8}$ in Enq. (7b) $A_{\gamma}=0.5108 \mathrm{~kg}^{1 / 2} \cdot \mathrm{mol}^{-1 / 2}$ at $298.15 \mathrm{~K}$, and $A_{\Phi}=0.392 \mathrm{~kg}^{1 / 2} \cdot \mathrm{mol}^{-1 / 2} \mathrm{De}-$ bye-Hückel parameters; $b=1.2 \mathrm{~kg}^{1 / 2} \cdot \mathrm{mol}^{-1 / 2}$ constant equal for all electrolytes. ${ }^{[35-37]}$

The other constants and variables are $N_{A}$ : Avogadro constant, $d_{\mathrm{w}}$ : density $\left(\mathrm{kg} \cdot \mathrm{m}^{-3}\right)$ of solvent water at temperature $T, e$ : charge of electron, $\varepsilon_{0}$ : electric permittivity of vacuum, $\varepsilon_{\mathrm{w}}$ : dielectric constant of water at temperature $T, k$ : Boltzmann constant, $T$ : absolute temperature.

The cation-anion interaction coefficients (Eqns 7c, 7d, 7e) used to describe the non-ideal behaviour and the triple ion interaction coefficients are given, with $\beta_{i j}{ }^{(0)}, \beta_{i j}{ }^{(1)}, \beta_{i j}{ }^{(2)}, \alpha_{1}, \alpha_{2}$ and $C_{i j}{ }^{\phi}$ from literature tables or from the Pitzer database. ${ }^{[38]}$

The parameters $\beta_{i j}^{(0)}, \beta_{i j}^{(1)}$ and $\beta_{i j}^{(2)}$ are specific for each electrolyte. They are obtained through fitting of isothermal or isobaric thermodynamic data available for aqueous solutions of the considered electrolyte. The coefficient $\beta_{i,}^{(2)}$ is needed only for two-two (e.g. $\left.\mathrm{Mg}^{2+} \mathrm{SO}_{4}^{2-}\right)$ or higher valence electrolytes, in which the formation of electrostatic ion pairs is important. For solutions containing at least a univalent ion, $\alpha_{1}=1.2 \mathrm{~kg}^{1 / 2} \cdot \mathrm{mol}^{-1 / 2}$ and $\alpha_{2}=0 \mathrm{~kg}^{1 / 2} \cdot \mathrm{mol}^{-1 / 2}$, but for two-two electrolytes at $25{ }^{\circ} \mathrm{C}, \alpha_{1}$ $=1.4 \mathrm{~kg}^{1 / 2} \cdot \mathrm{mol}^{-1 / 2}$ and $\alpha_{2}=1.2 \mathrm{~kg}^{1 / 2} \cdot \mathrm{mol}^{-1 / 2}$. These values are often assumed to be independent of the temperature $T$ and the pressure $p$. $^{[39]}$

\section{Results: Chemical Ion Activity Measurements in Mixed Electrolyte Solutions}

In chemical thermodynamics, the activity $a$ represents the effective concentration or molality of a species in a mixture. The activity of an ion is greatly influenced by other constituents of the mixture. The difference between activity and other sample composition measurements is caused by the interaction between the molecules in non-ideal gases or solutions, either to attract or to repel each other. In practice, concentrations are often used instead of activities, that should be used to define equilibrium constants or reaction rates. There are

\subsubsection{Determination of the Nernst Sensitivity}

On the example showed in section 4.1.1, we have performed identical EMF measurements for all the membranes in Table 2. The 13 mixed electrolyte standard solutions each containing the five ions were pumped sequentially into the system for $1 \mathrm{~h}$ potentiometric measurements, and runs of five cycles were performed to assess the system stability. For each cycle of the standard solutions, the EMF values obtained with the ion-selective electrodes were collected and plotted versus the logarithm of the corresponding ion activity $\left(\log \left(a_{i} / m^{0}\right)\right)$ shown in Table 3 .

As seen in Fig. 3a and Fig. 3b, the membranes selective to the monovalent ions of sodium (ionophore $\mathrm{X}$ ) and potassium (ionophore I) yielded slopes of 61.9 resp. $57.2 \mathrm{mV}$ per decade at $\mathrm{t}=19.5^{\circ} \mathrm{C}$. These values are satisfyingly close to the Nernstian behaviour expected of $58.4 \mathrm{mV}$, showing a successful discrimination of the interfering ions present in the standard solutions. The $s$ values in the graphics are calculated using the linear fitting 

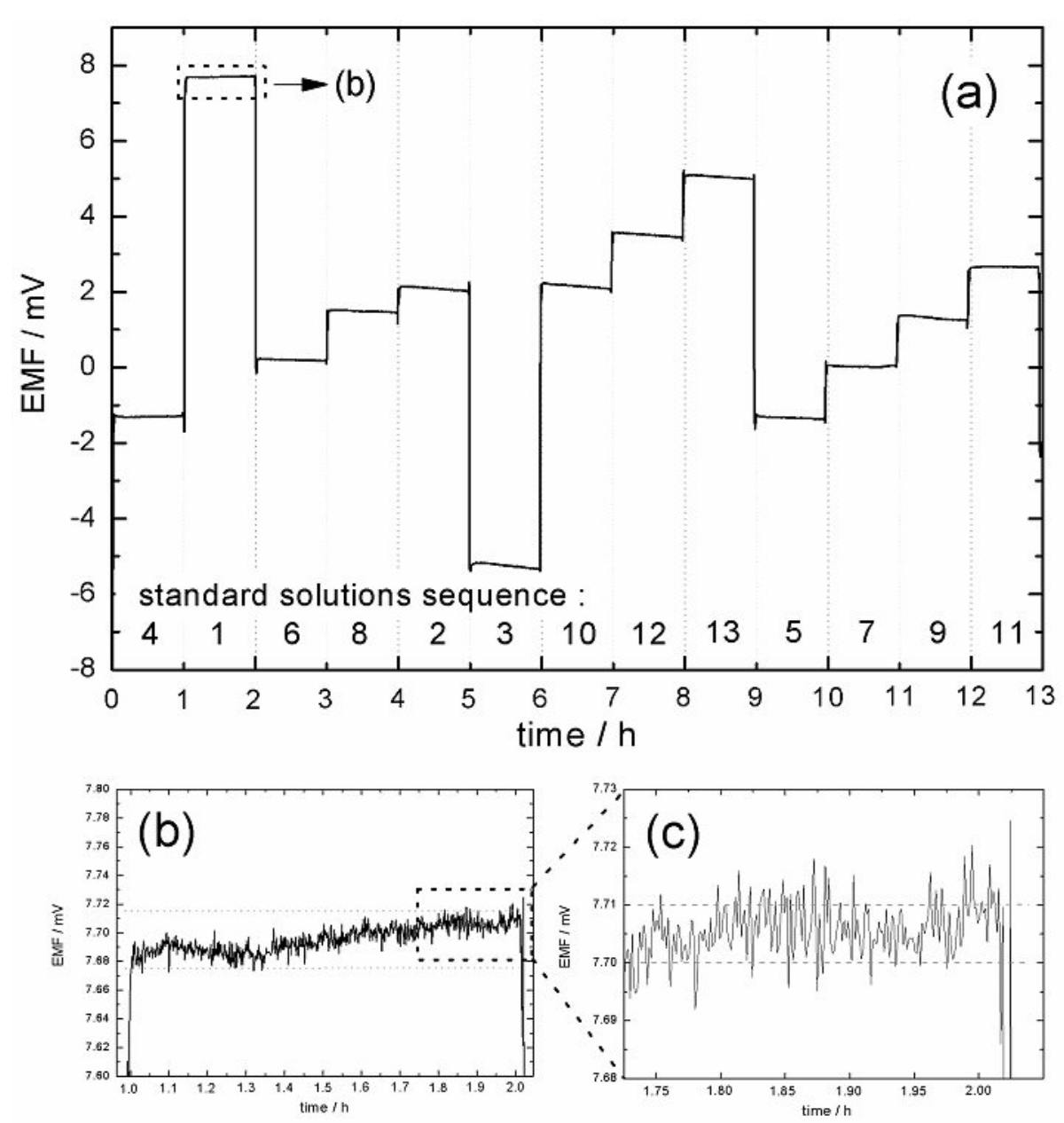

Fig. 2. Potentiometric measurements of standard solution sequences using the ion-selective membrane for $\mathrm{K}^{+}$(ionophore I). Fig. 2a shows the evolution of the potentiometric signal during one hour steady state measurement for each solution. Fig. $2 \mathrm{~b}$ shows the signal drift of $40 \mu \mathrm{V}$ during the measurement of solution. Fig. $2 \mathrm{c}$ shows the signal noise of $10 \mu \mathrm{V}$ in the last minutes of measurement of solution 1 .

tool of software Origin Pro 8 (OriginLab Corporation, Northampton, U.S.A.), representing the fitted standard deviation of the experimental linear slope values. In Fig. $3 \mathrm{c}$, the slope of $-46.2 \mathrm{mV}$ obtained for the chloride ISE (TDMACl), although different from the expected value $-58.4 \mathrm{mV}$ at $19.5^{\circ} \mathrm{C}$ clearly allows the detection of the selected ion from the interfering ions as well.

The two ISE used for calcium (ionophore I) and magnesium (ionophore VI) yielded slopes of $26.4 \mathrm{mV}$ resp. $25.1 \mathrm{mV}$, values that are certainly lower than the ideal expected $29.6 \mathrm{mV}$. The $\mathrm{Ca}^{2+}$ ions present in the mixture interfere with the $\mathrm{Mg} / \mathrm{Ca}$ selective electrode signal, but the membrane discrimination is high enough for this influence to be neglected. The slopes obtained are the result of measuring lower activity values for the electrolytes $\mathrm{Ca}^{2+}$ and $\mathrm{Mg}^{2+}$ in the standard solution mixture (Table 3$)$. In consequence, the $\left(\log \left(a_{\mathrm{i}} / m^{0}\right)\right)$ in Fig. 3d and Fig. 3e is slightly shifted to the non-linear domain of the activity scale, resulting in a lower slope. This effect being inherent to the activity scale of the target analyte, and in this case not related to the membrane or ionophore properties, the obtained values are suitable for a correct ISE detection of divalent ions.

\subsubsection{Determination of the Ion Activity in Test Solutions}

An experiment was performed to determine the potassium activity in three mixed electrolyte test solutions by calibration with three reference standard solutions, using the $\mathrm{K}^{+}$-selective membrane containing potassium ionophore I. At first, a three-point calibration curve was obtained as described earlier in this work, using minimum and maximum reference solution concentrations bracketing those of the samples. The slope obtained is $56.34 \mathrm{mV}$ $(\mathrm{s}=0.005 \mathrm{mV})$ and the intercept of 137.49 $\mathrm{mV}(\mathrm{s}=0.013 \mathrm{mV}$ ) (Fig. 4). The depicted analysis function of $E$ versus $\left(\log \left(a_{i} / m^{0}\right)\right)$ (Eqn. (8), Fig. 4, full line) is given with the confidence interval of $95 \%$ (dotted lines), and the unknown potassium activity of the test samples can be mathematically deter- mined, as well as their respective uncertainties.

$$
a_{i}=10^{\frac{E M F \text {-intercept }}{\text { slope }}}
$$

The results obtained from Fig. 4 for the three samples are shown in Table 4, where the normally unknown values for the molality and the activity of $\mathrm{K}^{+}$(calculated according to the Pitzer model) are shown. With the EMF values obtained experimentally, the logarithm of the $\mathrm{K}^{+}$activity is traced back with its related uncertainty. The results obtained are consistent, since the experimentally determined activity is comparable to the values determined with the model.

\section{Conclusions}

Based on metrological principles an ion-selective measurement procedure for the determination of the chemical activity of ions in mixed electrolyte solutions has been proposed. The traceability of the results is achieved by a chain of comparisons of all parameters involved. A convention for their activities similar to measurements in $\mathrm{pH}$ has been adopted. ${ }^{[40]}$ The ion activity approach of Pitzer is fully suitable to measure activities in complex electrolyte mixtures. The most relevant steps involved have been carefully studied to set the best conditions and therefore achieving the lowest possible uncertainties, resulting in the smallest impact on the combined uncertainty.

Building the activity scale involves the collection of two main data flows: On one hand the determination of the activity of the electrolyte solutions is performed, where the influence parameters that are taken into account are the gravimetric data, the hardware specifications, the purity factors and the air buoyancy. On the other hand the acquisition of the potentiometric signal must be set up, where the contributions of the ion-selective membrane, the diffusion potential, the inner electrolyte and the measuring device have a considerable influence on the potential stability, drift and signal noise.

The results presented in this work demonstrate the validity of the method for the determination of ion activity by potentiometry with traceable data. For 13 standard reference solutions each containing five ions of interest, namely $\mathrm{Na}^{+}, \mathrm{K}^{+}, \mathrm{Ca}^{2+}, \mathrm{Mg}^{2+}$ and $\mathrm{Cl}^{-}$, the Nernst sensitivities were successfully evaluated with a selection of ionselective membranes. The results achieved are close to the expected calculated values. The corresponding activity scales were plotted and an assay was performed to de- 


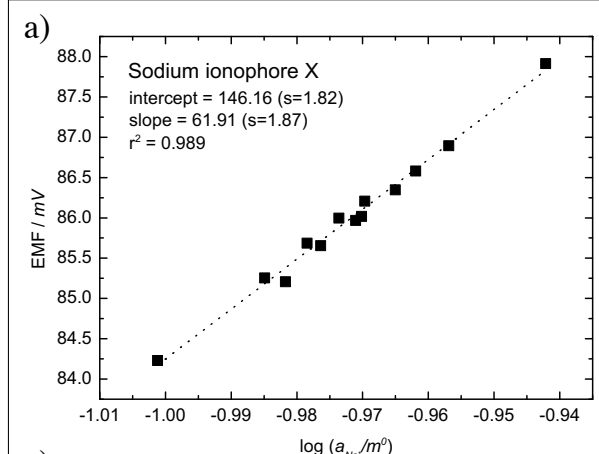

c)
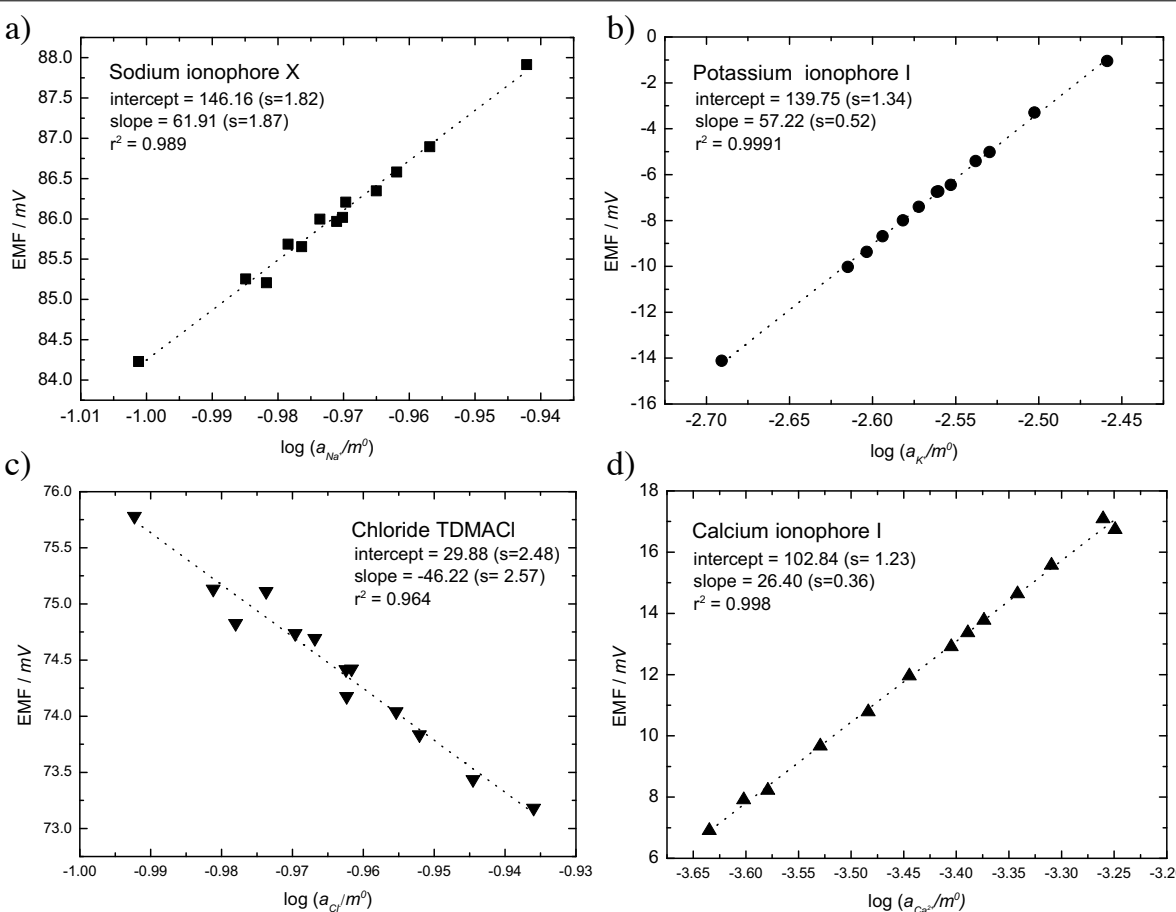

e)

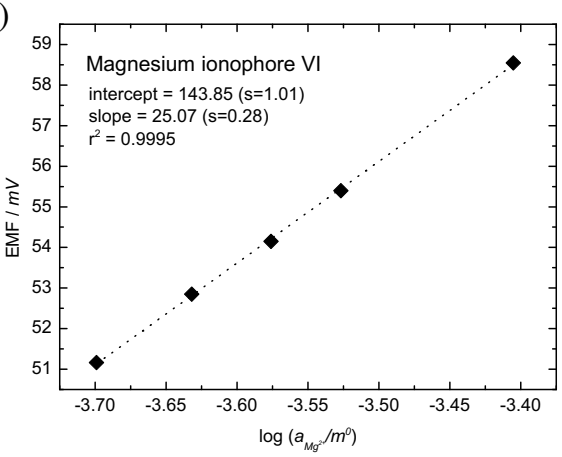

d)

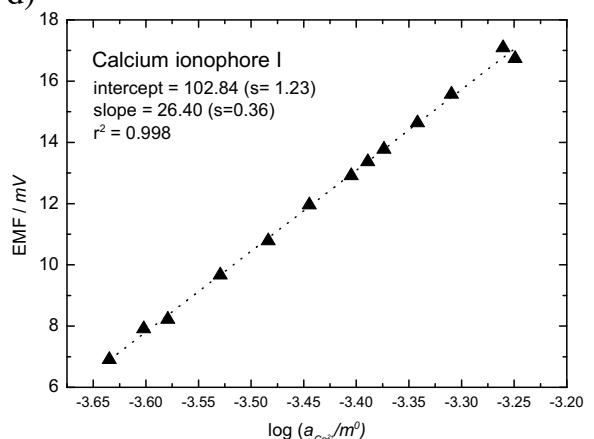

f)

\begin{tabular}{lc}
$\begin{array}{c}\text { Ion-selective } \\
\text { membrane }\end{array}$ & $\begin{array}{c}\text { Nernst sensitivity } \\
(\mathbf{m V})\end{array}$ \\
\hline $\mathrm{Na}^{+}(X)$ & $61.9(s=1.9)$ \\
$\mathrm{K}^{+}(I)$ & $57.2(s=0.5)$ \\
$\mathrm{Cl}^{-}(T D M A C l)$ & $-46.2(s=2.6)$ \\
$\mathrm{Ca}^{2+}(I)$ & $26.4(s=0.4)$ \\
$\mathrm{Mg}^{2+}(V I)$ & $25.1(s=0.3)$ \\
\hline & $\left(t_{\text {cell }}=19.5^{\circ} \mathrm{C}\right)$
\end{tabular}

Analytical Chemistry Section) are very much thanked for their technical help in the laboratory. For the permanent support in mechanics and electronics we thank both teams for their great help namely Thomas Krebs, Andreas Hurni, Roland Siffert, Erich Moll, Beat Zwahlen, Martin Rüfenacht and Rolf Zwahlen. Additional thanks go to Thomas Pulfer for the potentiometer calibration and to Alexander Tschudin and Johanna Saner for the calibration of the temperature sensors. The valuable support of Caspar Demuth and Jürg Müller (both ZHAW, Wädenswil, Switzerland) as well as Stefan Spichiger (C-CIT, Wädenswil) is much appreciated.

Received: June 29, 2009

[1] A. Menditto, M. Patriarca, 'Diagnostic test: Principles of Validation and Quality', 2008, 08, 43.

[2] S. Wunderli, H. Andres, Electroanalysis 2008, $20,324$.

[3] Off. J. Europ. Communities, Directive 98/79/ EC of the European Parliament and the Council on In Vitro Diagnostic Medical Devices 1998, L331, 1 .

[4] P. DeBièvre, H. Günzler, 'Traceability in Chemical Measurement', 2005, p 177, Springer, Berlin.

[5] E. Völkert, 'Implementation of Traceability Needs and Perspective of the In-Vitro-Diagnosticum Industry', 2004, 9, 237.

[6] 'Laboratory medicine - Requirements for Reference Measurement Laboratories', ISO 15195: 2003, 1.

[7] Quality Management System Fundamentals and Vocabulary', ISO 9000: 2005.

[8] 'General Requirements for the Competence of Testing and Calibration Laboratories', ISO/IEC 17025: 2005, 1.

[9] 'Medical Laboratories - Particular Requirements for Quality and Competence', ISO 15189: 2007.

[10] R. Dybkaer, Accred. Qual. Assur. 1999, 4, 401.

[11] International Organization for Standardization, 'Guide to the Expression of Uncertainty in Measurement', 1995, p. 1, officially available on internet free of charge with minor correc- termine the activity of unknown test sample solutions with a three point calibration curve. The analysis function used to trace these values back gave satisfying results with relative uncertainties in the percent range for the activities.

\section{Acknowledgements}

We are indebted to the project initiators Ursula Spichiger (Wädenswil, formerly at ETH) and Ulrich Feller (METAS). The research within this EURAMET joint research project Tracebioactivity receives funding from the European Community's Seventh Framework Programme, ERA-NET Plus, under the iMERA-Plus Project - Grant Agreement No. 217257. Our collaborators within work package 3 of the project Tracebioactivity: Petra Spitzer (PTB, Germany), Michal Mariàssy (SMU, Slovakia), Beatrice Adel and Frank Bastkowski (both PTB, Germany) are acknowledged as well as the work of the project coordinator Bernd Güttler (PTB). For the software programming we thank Heinz Herren (ISET GmbH, Laupen, Switzerland). Olivier Brunschwig and Daniel Schwaller (METAS,

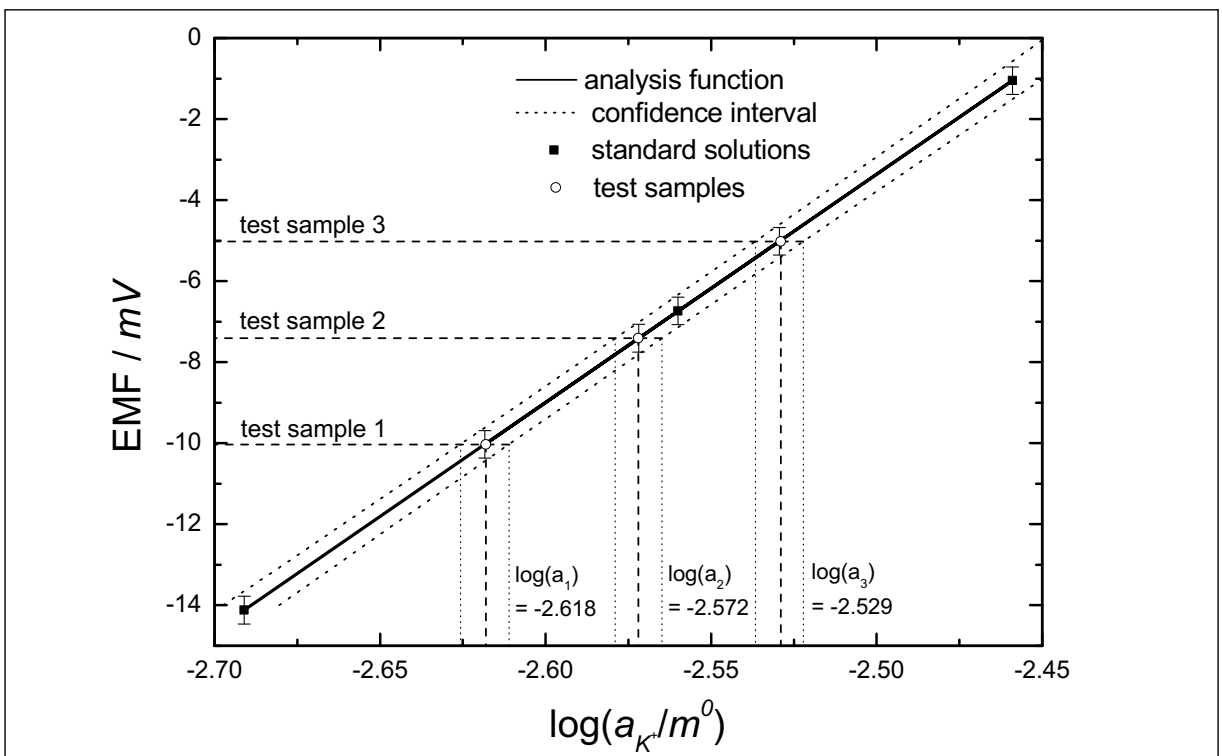

Fig. 4. Determination of the activity of potassium of electrolyte mixtures (using an ion-selective membrane $\mathrm{K}^{+}$ionophore I). The EMF values obtained by potentiometric measurements are introduced in the analysis function to trace back the logarithm of the activity of the $\mathrm{K}^{+}$content in the sample solutions. 
Table 4. Determination of the potassium activity in three test samples with a three point calibration curve

\begin{tabular}{|c|c|c|c|c|c|c|c|}
\hline Sample & $\begin{array}{l}\mathrm{K}^{+} \text {molality } \\
/(\mathbf{m m o l} \cdot \\
\left.\mathbf{k g}^{-1}\right)\end{array}$ & $\begin{array}{l}\text { Activity } \\
\text { (Pitzer) } \\
/\left(\mathrm{mmol}^{-1}\right. \\
\left.\mathbf{k g}^{-1}\right)\end{array}$ & $\begin{array}{l}\text { EMF } \\
/ \mathrm{mV}\end{array}$ & $\begin{array}{l}\log \left(a_{k+}\right) \\
\text { exp. }\end{array}$ & $u(\log (a))$ & $\begin{array}{l}\text { Activity a } \\
\text { (exp). } \\
\text { /(mmol- } \\
\text { kg }^{-1} \text { ) }\end{array}$ & $\begin{array}{l}\mathrm{u}(\mathrm{a}) \\
/(\mathrm{mmol} \\
\left.\mathrm{kg}^{-1}\right)\end{array}$ \\
\hline 1 & 3.23 & 2.427 & -10.030 & -2.618 & 0.007 & 2.410 & 0.041 \\
\hline 2 & 3.58 & 2.678 & -7.408 & -2.572 & 0.007 & 2.679 & 0.044 \\
\hline 3 & 3.96 & 2.954 & -5.018 & -2.529 & 0.007 & 2.958 & 0.049 \\
\hline
\end{tabular}

tions and restrictions to use under: Working Group 1 of the Joint Committee for Guides in Metrology (JCGM/WG 1) JCGM 100: 2008 http://www.bipm.org/utils/common/documents/ jcgm/JCGM_100_2008_E.pdf.

[12] K. Stinshoff, AACC Annual Meeting, 2003.

[13] 'In Vitro Diagnostics Medical Devices Measurement of Quantities in Biological Samples - Metrological Traceability of Values Assigned to Calibrators and Control Materials', EN ISO 17511: 2003

[14] Joint Committee for Traceability in Laboratory Medicine, http://www.bipm.org/jctlm/.

[15] R. Eugster, B. Rusterholz, A. Schmid, U. E. Spichiger, W. Simon, Clin. Chem. 1993, 39, 855.

[16] D. A. Skoog, F. J. Holler, T. A. Nieman, 'Principles of Instrumental Analysis', 1998, De Boeck, Paris.

[17] H. Kaden, W. Vonau, J. Prakt. Chem. 1998 $340,710$.

[18] R. H. Stokes in 'Activity Coefficients in Electrolyte Solutions', Ed. K. S. Pitzer, 2nd ed., CRC Press, 1991, chapter 1, p.1.

[19] F. Deyhimi, Z. Karimzadeh, R. SalamatAhangari, Fluid Phase Eq. 2007, 254, 18.

[20] K. S. Pitzer, J. Phys. Chem. 1973, 77, 268.

[21] K. S. Pitzer, G. Mayorga, J. Phys Chem. 1973, 77, 2300 .

[22] K. S. Pitzer, G. Mayorga, J. Sol. Chem. 1974, 3, 539.

[23] K. S. Pitzer, J. J. Kim, J. Am. Chem. Soc. 1974, 96, 5701.

[24] D. A. MacInnes, J. Am. Chem. Soc. 1919, 41, 1086.

[25] A. Reichmuth, S. Wunderli, M. Weber, V.R. Meyer, Microchim. Acta 2004, 148, 133 .

[26] E. Bakker, Anal. Chem. 1997, 69, 1061.
[27] M. Nägele, E. Bakker, E. Pretsch, Anal. Chem. 1999, 71, 1041

[28] B. P. Nikolskij, M. Schulz, A. Belijustin in 'Glass Electrodes for Hydrogen and Other Cations', Ed. G. Eisenmann, Marcel Dekker, New York, 1967.

[29] P. Henderson, Z. Phys. Chem. 1907, 59, 118

[30] A. Dybko, Sensors 2001, 1, 29.

[31] V. Rumenjak, I. Kruhak, S. Milardovi, Clin. Chim. Acta 2003, 335, 75 .

[32] G. Zhang, N. Spycher, E. Sonnenthal, C. Steefel, Proceedings of TOUGH Symposium 2006, 1.

[33] S. L. Clegg, M. Whitfield in 'Activity Coefficients in Electrolyte Solution', Ed. K. S. Pitzer, 2nd ed., CRC Press, 1991, chapter 6, p. 279.

[34] K. S. Pitzer, Activity Coefficients in Electrolytes Solutions, Ed. K. S. Pitzer, 2nd ed., CRC Press, 1991, chapter 3, p. 75.

[35] M. Luckas, J. Krissmann, 'Thermodynamik der Elektrolytlösungen', Springer, 2001, p. 136.

[36] U. E. Spichiger-Keller, 'Chemical Sensors and Biosensors for Medical and Biological Applications', Wiley-VCH, Weinheim, 1998, p. 120.

[37] L. F. Sylvester, K. S. Pitzer, J. Phys. Chem. 1977, 81, 1822.

[38] G. M. Rosenblatt, AIChE Journal 1981, 619.

[39] L. Marini, Developments in Geochemistry 2006, 11, 69.

[40] R. P. Buck, S. Rondinini, F. G. K. Baucke, C. M. A. Brett, M. F. Camões, A. K. Covington, M. J. T. Milton, T. Mussini, R. Naumann, K. W. Pratt, P. Spitzer, G. S. Wilson, Pure Appl. Chem. 2002, 74, 2169 . 